\title{
Suicide literacy, suicide stigma, and psychological help seeking attitudes among Arab youth
}

\author{
Yasmin Al-Shannaq $^{1}$ (D) Mohammed Aldalaykeh $^{2}$ \\ Accepted: 14 June 2021 / Published online: 20 June 2021 \\ (C) The Author(s), under exclusive licence to Springer Science+Business Media, LLC, part of Springer Nature 2021
}

\begin{abstract}
To date, very little is known about the current situation of suicide and its effects on psychological help-seeking attitudes in Arab countries. Therefore, this study aimed to assess the levels of suicide literacy and suicide stigma and their relationships with attitudes towards seeking psychological help among a sample of Arab youth in Jordan. A descriptive, correlational, crosssectional study was conducted using an anonymous online self-report survey to collect data. This study included a total of 707 Arab youth aged $16-24$ years (mean $=18.60, \mathrm{SD}=1.95)$. Most of the participants were male $(n=398,56.3 \%)$. The participants were found to have very low suicide literacy levels, relatively high suicide stigma levels, and negative attitudes towards seeking psychological help. Family members, relatives, and friends were the most frequently reported sources of psychological help, while mental healthcare professionals were the least frequently reported sources. A significant negative correlation was found between attitudes towards seeking psychological help and both stigmatization and glorification subscales of suicide stigma. While a significant positive correlation was found between attitudes towards seeking psychological help and suicide literacy. The predictors of attitudes towards seeking psychological help were gender, suicide literacy, and glorification of suicide stigma among Arab youth. The results of this study confirm the presence of an urgent need for designing and implementing educational programs and awareness campaigns on suicide and mental health among youth in schools and universities. Such interventions may increase knowledge, decrease stigma, change psychological help-seeking attitudes, and hence improve the use of mental health services and may prevent suicide among this age group.
\end{abstract}

Keywords Suicide $\cdot$ Stigma $\cdot$ Attitudes $\cdot$ Youth $\cdot$ Arab $\cdot$ Jordan $\cdot$ Mental health

\section{Introduction}

Every year, about 800,000 people around the world die from suicide; every $40 \mathrm{~s}$, one person commits suicide according to the World Health Organization (WHO) (WHO, 2019). Over $79 \%$ of these suicide cases occur in developing countries (WHO, 2019). Globally, suicide is considered the second leading cause of death among people aged 15-29 years (WHO, 2019), and suicide rates are much higher among people aged 16-24 than among other age groups (Madge et al.,

Yasmin Al-Shannaq

ymshannaq@just.edu.jo

1 Community and Mental Health Nursing Department, Faculty of Nursing, Jordan University of Science and Technology, Irbid, Jordan

2 Community and Mental Health Nursing Department, Faculty of Nursing, Jordan University of Science and Technology, Irbid, Jordan
2008). The onset of mental health problems usually occurs during adolescence (Rockett et al., 2012; WHO, 2019). Unfortunately, most cases of mental health problems, such as depression, are often unrecognized, misdiagnosed, or untreated (Rockett et al., 2012; WHO, 2019). Consequently, one of the most common effects of the nontreatment or late detection of mental health problems is the increased risk of suicide (Arafat et al., 2019; Choudhry et al., 2016). Stigma towards mental illness and towards suicidality in particular is considered one of the most common barriers for seeking professional psychological help (Clement et al., 2015; Keller et al., 2019; Niederkrotenthaler et al., 2014). In addition, knowledge about suicide is inadequate among youth and has not been widely explored (Ram et al., 2017). Therefore, stigma and lack of knowledge on suicide may limit the development and implementation of effective suicide prevention strategies and healthcare interventions at the individual and community levels (Niederkrotenthaler et al., 2014). The evidence shows that increasing mental health literacy and lowering stigma 
towards suicidal persons are correlated with more positive attitudes towards seeking professional psychological help for mental health problems (Peel et al., 2017).

Although interest in suicide research has been increasing in recent years (Pandey et al., 2019), the situation of suicide in developing countries, including countries in the Middle East region, has been largely ignored (Mahfoud et al., 2011). Although suicide incidence is low among children and adolescents in the Arab region, it has been increasing over the past decade (Morad et al., 2005). Many Arab countries have recently been exposed to wars, terrorism, and social and economic conflicts, which may have played a critical role in the development of behavioral and mental problems and illnesses (e.g., suicide) among Arab populations (El Halabi et al., 2020; Okasha et al., 2012). Thus, in comparison to other than age groups, Arab youth are at higher risk of developing mental illnesses and, hence, of suicide. This highlights the presence of an urgent need to develop and support suicide and mental illness polices and interventions in developing countries (El Halabi et al., 2020; Liu et al., 2018).

\section{Suicide in Jordan: An Exemplar of Arab and Muslim Countries}

Jordan is an Arab country located in the Middle East region. The country's official language is Arabic. The majority of the Jordanian population are Muslim. Muslim is an Arabic word that refers to the followers of the religion of Islam. In Jordan, the suicide rate among the 15-29 age group is 3.2 per 100,000 for both sexes, 3.8 per 100,000 for males, and 2.5 per 100,000 for females (WHO, 2018). Results from the 2007 Global School Health Survey indicated that $16.6 \%$ of Jordanian adolescents had previously experienced suicidal thoughts (Mahfoud et al., 2011). Previous studies have found that the prevalence of mental illnesses in Jordan is higher among adolescents and youth (i.e. school and university students) than among other age groups, which may indicate an increased risk of suicide among the former (Aldalaykeh et al., 2019; Dardas et al., 2019; Ismayilova et al., 2013). Dardas et al. (2019) reported that about $34 \%$ of Jordanian adolescents had moderate to severe levels of depression, and among these adolescents, $57 \%$ reported that they would choose to seek help from a family member. Another study found that about $57.7 \%$ of Jordanian university students had mild to moderate levels of depression and among these students, $76 \%$ reported religious practices as being their preferred help-seeking behavior, followed by talking with family and friends (44.8\%) (Aldalaykeh et al., 2019). Only one recent study reported that Jordanian university students had low levels of suicide literacy and high levels of stigma towards suicide (Aldalaykeh et al., 2020). Therefore, it is vital to investigate the phenomena of suicide and its effects on psychological help-seeking attitudes among Arab youth, as they are considered to be a vulnerable population at a high risk of suicide. To the best of our knowledge, no studies conducted in Arabic countries like Jordan have investigated suicide and its effects on psychological help-seeking attitudes among Arab youth, indicating a gap in the literature. Therefore, this study aimed to assess the levels of suicide literacy and suicide stigma and their relationships with attitudes towards seeking psychological help among a sample of Arab youth in Jordan. Despite the crosssectional nature of this study, this study informs the current literature with important evidence on the prevalence of suicide literacy, suicide stigma, and attitudes towards seeking psychological help and their relationships among a sample of Arab youth in Jordan.

\section{Arabic and Islamic Cultural Perspectives on Mental Illness and Suicide}

Despite Arab societies being widely diverse, Arabs share many mental health beliefs, values, and practices which shape the ways they perceive and manage mental illnesses, including suicide. Islam encourages its followers to seek healthcare and to accept and treat their illnesses in order to improve their mental health and maintain their overall well-being (Koenig \& Al Shohaib, 2019). The evidence indicates that religion plays an integral role in the lives of Muslims (Al-Shannaq et al., 2021; Amiri et al., 2013; Eskin et al., 2020; Shah \& Chandia, 2010). Muslims use their religious faith, beliefs, and practices to cope and deal with life stressors and events in their lives, including illness (AlShannaq et al., 2021; Eskin et al., 2020; Koenig \& Al Shohaib, 2019). However, Muslims strongly believe that only Allah (God) is primarily responsible for everything, including illness (Ciftci et al., 2013). They also believe that mental illness as a test or punishment from Allah in order to make them more connected to Him and enlightens their mistakes (Ciftci et al., 2013). Muslims may combat mental illness by, for example, reading and reciting the Holy Quran, thanking and praying to Allah, making supplication to Allah, and showing patience and tolerance. Although many Muslims show positive attitudes towards mental illness, stigma regarding mental illness remains widely existent within Arab societies (Amri \& Bemak, 2013; Ciftci et al., 2013; Koenig \& Al Shohaib, 2019). Arab Muslims suffers from stigma towards mental illness and its treatment like other populations. Stigma is considered a major barrier for individuals with mental illness to seek professional healthcare and lead healthy lives (Amri \& Bemak, 2013; Ciftci et al., 2013; Koenig \& Al Shohaib, 2019). This has often been attributed to Arab culture's collectivistic nature and emphasis on strong family ties (Dardas \& Simmons, 2015; Koenig \& Al Shohaib, 2019; Zolezzi et al., 2017), which encourages individuals to prioritize the needs and problems of others over their own. Thus, in Arab culture, mental illness is not left to the ill individual to handle alone; rather it is often handled as a family issue, with the ill person's family members and relatives forming a support network to help overcome the mental 
illness. Stigma towards mental illness is not only faced by mentally ill people, but it is also faced by their family members (Amri \& Bemak, 2013; El Halabi et al., 2020; Koenig \& Al Shohaib, 2019). Further, Arab Muslims may often feel the need to hide their diagnosis with mental illness and treatment from others, in addition to keeping discussions about their mental health problems private within their families in order to remain accepted by society and protect themselves and their families from negative labels and public view (Amri \& Bemak, 2013; Dardas \& Simmons, 2015; Dardas et al., 2019; Koenig \& Al Shohaib, 2019; Rayan \& Jaradat, 2016; Zolezzi et al., 2017). Researchers and healthcare providers should become involved with families and communities, and not only with individuals, in order to address negative beliefs, attitudes, and behaviors towards mental illness and suicide (El Halabi et al., 2020).

Suicide is considered sinful behavior and is prohibited in the religion of Islam (Amiri et al., 2013; El Halabi et al., 2020; Eskin et al., 2019). Suicidal behaviors include suicidal ideations or thoughts, suicide attempts, and completed suicide that leads to ending one's life (Eskin et al., 2020). Although the incidence of suicide mortality is lower in Muslim countries than in non-Muslim countries, the incidence rates of suicidal ideations and attempts in Muslim countries is higher than in non-Muslim countries (Amiri et al., 2013; Eskin et al., 2019; Eskin et al., 2016). Religious beliefs, attitudes, and practices may also shape people's attitudes towards suicide, and thus, people with religious affiliation may view suicide differently than do people without religious affiliation (Amiri et al., 2013; El Halabi et al., 2020; Eskin et al., 2020). Religion may protect its followers from committing suicidal behaviors, including suicidal ideations (Eskin et al., 2019; Lester \& Walker, 2017), suicide attempts (Eskin et al., 2019; Lawrence et al., 2016), and completed suicide (Eskin et al., 2016). It may also be associated with lower rates of suicidal behaviors and decreased acceptability of suicide (Amiri et al., 2013; El Halabi et al., 2020; Eskin et al., 2020; Shah \& Chandia, 2010). The evidence shows mixed results regarding the association between religion and suicidal behaviors (Eskin et al., 2019; Eskin et al., 2020), with studies in Western and Middle Eastern countries reporting a significant inverse association between religion and suicide (Koenig et al., 2012; Koenig \& Al Shohaib, 2019; Shah \& Chandia, 2010; Wu et al., 2015). For example, in a recent study conducted in Tunisia by FekihRomdhane et al. (2020), a negative relationship between religion and suicidal ideations among university students was reported. The evidence on the relationship between religion and suicide is complex in nature, and therefore, it can be difficult to compare between Muslim and non-Muslim countries in this regard (Eskin et al., 2020). The variation in the findings of previous studies may be attributed to differences in sociodemographic characteristics, geographical regions, healthcare systems, wealth, economy, culture, religion, political systems, and suicide rates between different countries, as well as the use of different methodologies, designs, and sample sizes in different studies (Eskin et al., 2019; Eskin et al., 2020). However, the evidence indicates the need to address the stigma within Arab societies against individuals with mental illnesses in order to enhance access to mental health services, increase acceptance of mental illness at the community level, and improve the overall well-being of mentally ill people. The background and findings of the current study may provide some valuable information, particularly for those who are not familiar with Jordanian or Arab Islamic cultures, of the mental health issues (e.g., suicide) within these cultures. This may help researchers and healthcare providers address and understand mental health issues, such as suicide, in a way which meets Arab Muslims' multidimensional needs and concerns within a multidisciplinary approach.

\section{Theoretical Framework}

The Ecological Systems Theory (EST) provides the conceptual model for this current study (Bronfenbrenner, 1977). The model places the individual at its center, with other levels of systems nested around the individual (Tanhan, 2019; Tanhan, 2020). Individuals are continuously affected through their relationships with these systems within the context of their environment (Tanhan, 2019; Tanhan, 2020). The EST focuses on the multiple contextual factors in the individual's environment, including biopsychosocial, spiritual, political, and economic factors, based on a comprehensive and holistic approach (Tanhan \& Francisco, 2019). The model divides the individual's environment into five main systems/levels as follows: the microsystem (e.g., individuals' relationship with their parents), the mesosystem (e.g., the interactions between individuals' parents and their peers), the exosystem (e.g., parents' workplace and the social media), the macrosystem (geographic area and cultural and religious aspects), and the chronosystem (e.g., the COVID-19 pandemic) (Bronfenbrenner \& Evans, 2000). These levels comprise many factors which vary among individuals and affect them differently, in addition to changing over time following a dynamic process. These levels are interrelated and should be considered together (Tanhan \& Francisco, 2019; Tanhan \& Strack, 2020), with each level influencing the other and its impact depending on its relationship with other levels during the individual's life development (Tanhan \& Francisco, 2019). For example, individuals' knowledge, beliefs, and attitudes regarding mental health issues (e.g., suicide) may be influenced by others and the cultural and religious variables in their environments. Recent evidence indicates that the EST has been applied among different populations, particularly among Muslims (Tanhan, 2019; Tanhan \& Francisco, 2019; Tanhan \& Strack, 2020). The aim of this model is to guide the generation of qualitative and quantitative research questions that may lead to investigating ecological systems collectively 
to address mental health issues and improve the overall wellbeing of individuals at all levels. This may be achieved through using an online photovoice as a qualitative method, testing hypotheses, describing the relationships between the contextual factors of the ecological systems, or developing and evaluating the effectiveness of educational/clinical interventions/services in order to better understand mental health issues. Using this model, we assessed the sociodemographic characteristics, suicide literacy levels, suicide stigma levels, and attitudes towards seeking psychological help, in addition to their relationships, among a sample of Arab youths within the context of their environment in Jordan.

\section{Methods}

\section{Participants and Procedures}

This study used a descriptive, correlational, cross-sectional design. Data were collected between 10 June and 21 July 2020 using an anonymous online self-report survey. In this study, youth refers to individuals aged 15-24 years and includes middle and late adolescence based on the United Nation (UN) definition (UN, 2020). Thus, all Arab youth aged 16-24 years in Jordan were eligible to participate in this study. The study participants were recruited through social networking platforms (i.e. Facebook and WhatsApp). The snowball sampling method was also used by teachers and students to reach a larger number of respondents. Institutional review board (IRB) approval was obtained from the principle investigator's affiliated university. The validated Arabic versions of the standardized measures were used to assess the participants' demographics, suicide literacy, suicide stigma, and attitudes towards seeking professional psychological help. A Google Forms link to the anonymous online survey was shared with the potential participants through social media platforms. Prior to data collection, informed consent was obtained from the participants online. The participants were instructed to read the informed consent page at the beginning of the online survey and then click on the agreement button at the bottom of the page if they agreed to participate. After that, they were asked to complete the online self-report survey. The participants were informed about the study purpose, procedures, and use of data and assured that their participation was totally voluntary and that their identities would be kept confidential.

\section{Measures}

\section{Demographic Data}

A demographics questionnaire was used to collect data on the socio-demographic and clinical characteristics of the sample. The questionnaire included items related to age, gender, family size (i.e. number family members living in the same house), educational level and student/nonstudent, employment status, health insurance, employment status of parents, physical exercise, smoking, alcohol and substance use, presence of chronic physical or mental illnesses, history of suicidal thoughts or suicide attempts, family history of mental illnesses and suicidal thoughts or suicide attempts, and relationships with parents and others, including family members, relatives, and friends. The questionnaire also included two questions about previous seeking psychological help and preferred sources of help, which included choosing from the following: (1) family members, relatives, and friends, (2) general healthcare practitioners, (3) religious leaders, (4) mental healthcare professionals (e.g. psychiatrist or psychologist), and (5) no one.

\section{Suicide Literacy}

The Literacy of Suicide Scale (LOSS) was used to assess the participants' suicide literacy levels. The LOSS is a 12-item questionnaire used to assess knowledge about suicide, including suicide signs and symptoms ( 3 items), causes/nature (4 items), risk factors (3 items), and treatment and prevention (2 items) (Calear et al., 2014). Each item has two possible answers (i.e. true or false). The total score is obtained by summing the number of true answers and then converting the number into a percentage ranging from 0 to $100 \%$, with higher scores indicating higher suicide literacy levels. A validated Arabic version of this scale was used in this study (Aldalaykeh et al., 2020).

\section{Suicide Stigma}

The Stigma of Suicide Scale-short form (SOSS-SF) was used to assess the participants' suicide stigma levels. The SOSS-SF is a questionnaire used to measure stigma towards people who die by suicide in the community and comprises 16 items which describe people who have ended their life by suicide (Batterham et al., 2013a). The items are scored on a 5-point Likert scale ranging from $1=$ "strongly disagree" to $5=$ "strongly agree". The questionnaire includes three subscales: stigmatizing attitudes towards suicide ( 8 items), attribution of isolation or depression on suicide (4 items), and normalization or glorification of suicide (4 items). The score of each subscale is calculated by obtaining the respondent's mean score to the subscale's items. Each subscale's possible mean score ranges between one and five, with a mean score higher than three indicating agreement with the targeted concept. A validated Arabic version of this scale was used in this study (Aldalaykeh et al., 2020). In this study, the Cronbach's alpha for the SOSS-SF subscales were 0.76 for stigmatizing attitude toward suicide, 0.73 for attribution of isolation or depression on suicide, and 0.67 for normalization or glorification of suicide. 


\section{Psychological Help Seeking Attitudes}

The Attitudes Toward Seeking Professional Psychological Help Scale (ATSPPH) was used to assess the participants' psychological help-seeking attitudes. The ATSPPH is a 10-item short form measure used to assess attitudes towards seeking professional psychological help (i.e. seeking mental health treatments) among respondents (Fischer \& Farina, 1995). The items are scored on a 4-point Likert scale ranging from 0 (disagree) to 3 (agree). Items 2, 4, 8, 9, and 10 are reverse-scored. The total possible score ranges from 0 to 30 , and total scores are obtained by summing the responses to the 10 items. A higher score indicates more positive attitudes toward seeking psychological treatment. A validated Arabic version of this scale was used in this study (Dardas et al., 2016; Dardas et al., 2017). In this study, the Cronbach's alpha for the ATSPPH was 0.81 .

\section{Data Analysis}

The Statistical Package for the Social Sciences (SPSS, 23.0) was used to analyze the collected data. Descriptive statistics were used to describe: (a) the sample characteristics and (b) all of the major study concepts, including (1) suicide literacy, (2) suicide stigma, and (3) psychological help-seeking attitudes. An independent samples t-test was used to evaluate the differences in the participants' mean scores on the ATSPPH scale based on their sociodemographic and clinical characteristics categories. The Pearson product-moment correlation coefficient was used to examine the relationships between suicide literacy, suicide stigma, and psychological help-seeking attitudes among the sample. Hierarchical multiple regression was used to determine the predictors of psychological helpseeking attitudes among the sample.

\section{Results}

\section{Sample Characteristics}

Out of 715 potential participants, a total of 707 adolescents and youths completed the online survey and 8 refused to participate, leading to a response rate of $98.9 \%$. Table 1 presents the sociodemographic and clinical characteristics of the study participants. The participants' ages ranged from 16 to 24 years, with a mean age of 18.60 years $(\mathrm{SD}=1.95)$. The majority of the participants were male $(n=398,56.3 \%)$. The average family size was $6.10 \pm 1.51$ members, and the majority of the sample were students $(n=631,89.3 \%)$. More than half of the sample $(n=378,53.5 \%)$ were high school students, $28.3 \%(n=200)$ were bachelor's students, and 7.5\% $(n=53)$ were diploma students. Most of the participants $(n=535$, $75.7 \%)$ were unemployed, and $84.2 \%(n=595)$ had health insurance. Among the participants, $88.7 \%(n=627)$ reported that their fathers were employed, while $57.7 \%(n=408)$ reported that their mothers were unemployed. See Table 1.

The majority of the participants $(n=570,80.6 \%)$ did not practice physical exercise. Approximately half of the sample were cigarette smokers $(n=340,48.1 \%)$, while the majority did not have past or current experience with alcohol or drug use $(n=665,94.1 \%)$. In addition, most of the participants did not have chronic physical illnesses $(n=639,90.4 \%)$ or mental illnesses $(n=686,97.0 \%)$. Further, the majority of the participants reported no history of suicidal thoughts or suicide attempts $(n=631,89.3 \%)$. Most of the participants also reported no family history of mental illnesses $(n=662,93.6 \%)$ and no family history of suicidal thoughts or suicide attempts $(n=650,91.9 \%)$. The majority reported having good relationships with their parents $(n=551,77.9 \%)$ and with their family members, relatives, and friends $(n=580,82.0 \%)$. With regard to the first question related to seeking psychological help ("Have you ever sought psychological help?"), only 90 (12.7\%) participants reported having sought psychological help through mental health services in the past. As for the second question, "please specify your preferred source of psychological help when needed?", the most frequently reported sources of psychological help among the participants were family members, relatives, and friends $(n=$ $177,25 \%)$, followed by general healthcare practitioners $(n=$ $150,21.2 \%)$, no one $(n=135,19.1 \%)$, and religious leaders $(n=133,18.8 \%)$. On the other hand, the least reported source of psychological help was 'mental healthcare professionals' $(n=$ 112, 15.8\%). See Table 1.

\section{Suicide Literacy}

Table 2 presents the percentage of correct responses to the LOSS items. The participants' mean score in the LOSS was $3.82 \pm 2.13$. Only $158(22.3 \%)$ participants had a score higher than 6 out of 12, which reflects low suicide literacy levels among this sample. In general, the participants had difficulty answering the items correctly. None of the items had a percentage of correct answers over 50\%. Further, the participants had great difficulty answering items related to people with suicidal thoughts or ideations, and items related to the treatment or prevention of suicide. See Table 2.

\section{Suicide Stigma}

Table 3 depicts the descriptive statistics for the three subscales of the SOSS-SF. The participants' mean score in the stigma subscale was $3.24 \pm 0.74$, which reflects relatively high levels of stigma towards suicidal people. This is supported by the percentage of endorsement to the items that represent this scale. Six out of the eight items were endorsed by more than $50 \%$ of the sample, and the remaining two items (irresponsible and stupid) were endorsed by more than $40 \%$ of the sample. The participants' mean score in the isolation subscale was $3.63 \pm 0.74$, which 
Table 1 Sociodemographic and Clinical Characteristics $(N=707)$

\begin{tabular}{|c|c|c|c|}
\hline Variable & Category & Frequency $(\%)$ & $\begin{array}{l}\text { ATSPPH } \\
\text { t-test }\end{array}$ \\
\hline \multirow[t]{2}{*}{ Gender } & Female & $309(43.7)$ & $7.73 * * *$ \\
\hline & $\begin{array}{l}\text { Male } \\
\text { Yes }\end{array}$ & $\begin{array}{l}398(56.3) \\
631(89.3)\end{array}$ & $2.97 * *$ \\
\hline Employment status & $\begin{array}{l}\text { No } \\
\text { Yes }\end{array}$ & $\begin{array}{l}76(10.7) \\
172(24.3)\end{array}$ & 1.72 \\
\hline Health insurance & $\begin{array}{l}\text { No } \\
\text { Yes }\end{array}$ & $\begin{array}{l}535(75.7) \\
595(84.2)\end{array}$ & $3.56^{* * *}$ \\
\hline \multirow[t]{2}{*}{ Father's employment status } & $\begin{array}{l}\text { No } \\
\text { Yes }\end{array}$ & $\begin{array}{l}112(15.8) \\
627(88.7)\end{array}$ & 1.29 \\
\hline & $\begin{array}{l}\text { No } \\
\text { Yes }\end{array}$ & $\begin{array}{l}80(11.3) \\
299(42.3)\end{array}$ & $2.10 *$ \\
\hline Mother's employment status & $\begin{array}{l}\text { No } \\
\text { Yes }\end{array}$ & $\begin{array}{l}408(57.7) \\
137(19.4)\end{array}$ & -1.69 \\
\hline Physical exercise & $\begin{array}{l}\text { No } \\
\text { Yes }\end{array}$ & $\begin{array}{l}570(80.6) \\
340(48.1)\end{array}$ & 3.93 \\
\hline \multirow[t]{2}{*}{ Alcohol or substance use } & $\begin{array}{l}\text { No } \\
\text { Yes }\end{array}$ & $\begin{array}{c}367(51.9) \\
42(5.9)\end{array}$ & 0.84 \\
\hline & No & $665(94.1)$ & \\
\hline Chronic physical illness & Yes & $68(9.6)$ & -0.45 \\
\hline \multirow[t]{2}{*}{ Mental illness } & $\begin{array}{l}\text { No } \\
\text { Yes }\end{array}$ & $\begin{array}{c}639(90.4) \\
21(3.0)\end{array}$ & $-2.73 * *$ \\
\hline & No & $686(97.0)$ & \\
\hline \multirow[t]{2}{*}{ Family history of mental illness } & Yes & $45(6.4)$ & -0.03 \\
\hline & $\begin{array}{l}\text { No } \\
\text { Yes }\end{array}$ & $\begin{array}{r}662(93.6) \\
76(10.7)\end{array}$ & $-4.22 * * *$ \\
\hline Previous suicidal ideation or suicide attempt & No & $631(89.3)$ & \\
\hline \multirow[t]{2}{*}{ Family history of suicidal ideation or suicide attempt } & Yes & $57(8.1)$ & -1.07 \\
\hline & $\begin{array}{l}\text { No } \\
\text { Good }\end{array}$ & $\begin{array}{l}650(91.9) \\
551(77.9)\end{array}$ & -1.87 \\
\hline Relationship with parents & $\begin{array}{l}\text { Bad } \\
\text { Good }\end{array}$ & $\begin{array}{l}156(22.1) \\
580(82.0)\end{array}$ & 0.48 \\
\hline Relationship with others & Bad & $127(18.0)$ & \\
\hline \multirow[t]{2}{*}{ Previous psychological help seeking } & Yes & $90(12.7)$ & -5.92 \\
\hline & No & $617(87.3)$ & \\
\hline
\end{tabular}

$* P<0.05 ; * * P<0.01 ; * * * P<0.001$

ATSPPH: Attitudes towards seeking professional psychological help scale indicates that the participants strongly agreed that suicide is related to isolation. All of the items related to isolation were endorsed by more than $60 \%$ of the sample. The participants' mean score in the glorification subscale was $2.94 \pm 0.93$, which indicates that the participants disagreed that suicidal people should be glorified or normalized. Three items in the glorification subscale had an endorsement rate ranging between 40\%-45\%, and the remaining item (dictated) was endorsed by more than $23 \%$ of the sample. See Table 3.

\section{Correlations between Suicide Stigma, Suicide Literacy, and Psychological Help-Seeking Attitudes}

The Pearson product-moment correlation coefficient was used to examine the bivariate correlation between the major study concepts, including suicide literacy (LOSS), the three subscales of suicide stigma (SOSS$\mathrm{SF}$ ), and attitudes towards seeking professional psychological help (ATSPPH). A significant association was found between attitudes towards seeking psychological help and all of the major study concepts except for the SOSS-SF isolation subscale $(r=0.032, p>.05)$. A negative association was found between attitudes towards seeking psychological help and both the stigma and glorification subscales of the SOSS-SF $(r=-0.130, p<.01$ vs. $\mathrm{r}=-0.229, p<.001$, respectively). A significant positive correlation was found between attitudes towards seeking psychological help and the LOSS $(r=0.367$, $p<.001)$. Both the stigma and glorification subscales of the SOSS-SF had a significant negative correlation with 
Table 2 Correct Responses to Items from the Literacy of Suicide Scale (LOSS) $(\mathrm{N}=707)$

\begin{tabular}{ll}
\hline Item & $\begin{array}{l}\text { Percentage } \\
\text { correct }\end{array}$ \\
\hline There is a strong relationship between alcoholism and suicide (T) & 46.0 \\
Very few people have thoughts about suicide (F) & 44.3 \\
Not all people who attempt suicide plan their attempt in advance (T) & 43.1 \\
Men are more likely to die by suicide than women (T) & 42.3 \\
Seeing a psychiatrist or psychologist can help prevent someone from suicide (T) & 33.8 \\
A suicidal person will always be suicidal and entertain thoughts of suicide (F) & 32.4 \\
People who want to attempt suicide can change their mind quickly (T) & 31.7 \\
People who have thoughts about suicide should not tell others about it (F) & 27.7 \\
People who talk about suicide rarely kill themselves (F) & 23.6 \\
Most people who suicide are psychotic (F) & 22.9 \\
Talking about suicide always increases the risk of suicide (F) & 22.2 \\
If assessed by a psychiatrist, everyone who kills themselves would be diagnosed as depressed (F) & 12.4 \\
\hline
\end{tabular}

the $\operatorname{LOSS}(\mathrm{r}=-0.276, p<.001$ vs. $\mathrm{r}=-0.397, p<.001$, respectively). While no significant correlation was found between the isolation subscale of the SOSS-SF and the LOSS ( $r=-0.042, p>.05)$. Lastly, the stigma subscale of the SOSS-SF had a significant positive correlation with both the isolation and glorification subscales of the SOSS-SF $(r=0.462, p<.001$ vs. $r=0.608, p<.001$, respectively).

Table 3 Stigma of Suicide Scale-Short Form (SOSS-SF) Subscales and Item Responses $(\mathrm{N}=707)$

\begin{tabular}{llll}
\hline $\begin{array}{l}\text { Subscale } \\
\text { (Mean, SD) }\end{array}$ & Item & Agree/Strongly agree \% & Mean (SD) \\
\hline $\begin{array}{lll}\text { Stigmatization } \\
(3.24,0.74)\end{array}$ & Immoral & 55.7 & $3.22(1.12)$ \\
& Irresponsible & 48.6 & $3.39(1.01)$ \\
& Pathetic & 55.3 & $3.34(1.08)$ \\
& Stupid & 40.6 & $3.02(1.28)$ \\
& Coward & 57.5 & $3.10(1.25)$ \\
& Vengeful & 50.5 & $3.12(1.29)$ \\
& Shallow & 54.7 & $3.35(1.30)$ \\
& Embarrassment & 56.8 & $3.40(1.28)$ \\
Isolation & Lonely & 73.9 & $3.83(1.11)$ \\
$(3.63,0.74)$ & Isolated & 71.3 & $3.64(0.92)$ \\
& Disconnected & 60.1 & $3.35(1.02)$ \\
& Lost & 75.7 & $3.69(0.94)$ \\
Glorification & Nobel & 40.6 & $3.12(1.14)$ \\
$(2.94,0.93)$ & Strong & 42.2 & $2.88(1.31)$ \\
& Dedicated & 23.3 & $2.85(1.04)$ \\
& Brave & 45.4 & $2.90(1.69)$ \\
\hline
\end{tabular}

\section{Differences in Attitudes Towards Seeking Psychological Help Based on Sociodemographic and Clinical Variables}

The analysis revealed that the mean total ATSPPH score among all of the participants was $13.87(\mathrm{SD}=3.89)$, with scores ranging from 3 to 28 . These results indicated that the participants had negative attitudes towards seeking psychological help. Table 1 also shows the results of the t-test analysis, which examined the differences in the participants' mean ATSPPH scores based on sociodemographic and clinical variables. The analysis showed that female participants had more positive attitudes towards seeking psychological help in comparison to male participants (mean $15.11 \pm 4.49$ vs. mean $12.91 \pm 3.03, p<.001)$. In addition, participants who were not students had more positive attitudes compared to participants who were students (mean $15.12 \pm 3.63$ vs. mean 13.72 $\pm 3.72, p=.003$ ). Participants who did not have health insurance had more positive attitudes compared to participants who had health insurance (mean 15.06 \pm 3.52 vs. mean $13.65 \pm$ $3.92, p<.001)$. The analysis also showed that participants whose mothers were unemployed had more positive attitudes than participants whose mothers were employed (mean 14.13 \pm 3.98 vs. mean $13.52 \pm 3.75, p=.036$ ). Participants who had a mental illness showed significantly more positive attitudes towards seeking psychological help than participants who did not have a mental illness (mean $16.14 \pm 4.71$ vs. mean 13.80 $\pm 3.85, p=.007)$. Lastly, participants who had history of suicidal thoughts or suicide attempts showed significantly more positive attitudes towards seeking psychological help than participants who did not have history of suicidal thoughts or suicide attempts (mean $16.12 \pm 5.04$ vs. mean $13.60 \pm 3.64$, $p<.001)$. No significant differences in the mean ATSPPH 
scores of the participants based on other sociodemographic and clinical variables were found. See Table 1.

\section{Factors Predicting Attitudes Towards Seeking Psychological Help}

Hierarchical multiple regression analysis was performed using two steps to examine the factors influencing attitudes towards seeking psychological help, after including only the sociodemographic and clinical variables that showed significant effect on the participants' ATSPPH scores (Table 4). Also, the SOSS-SF isolation subscale was not included in the regression analysis, as it was not found to be significantly associated with attitudes towards seeking psychological help. The regression analysis included nine predictors as follows: LOSS, SOSS-SF stigmatization, SOSS-SF glorification, gender, being a student, health insurance, mother's employment status, having a mental illness, and history of suicidal thoughts or suicide attempts. The first step included only LOSS, SOSSSF stigmatization, and SOSS-SF glorification. This model was significant $(\mathrm{F}=39.31, p<.001)$, with an explained variance reaching $14.0 \%$. The LOSS was found to have the strongest significant independent effect, followed by SOSS-SF glorification; meanwhile, SOSS-SF stigmatization was not found to have a significant independent effect. The second step included all nine predictors and was also found to be significant $(\mathrm{F}=19.10, p<.001)$. The second step improved prediction significantly ( $\mathrm{F}$ change $=7.85, p<.001$ ) and showed an explained variance equal to $18.8 \%$. The strongest predictor was again the LOSS $(\beta=0.271)$, followed by gender $(\beta=-0.206)$ and SOSS-SF glorification $(\beta=-0.109)$. The remaining six predictors showed a nonsignificant independent effect. See Table 4.

\section{Discussion}

To date, very little is known about the current situation of suicide and its effects on psychological help-seeking attitudes in Arab countries like Jordan. This study was to investigate suicide stigma, suicide literacy, and attitudes toward seeking professional psychological help among a sample of Arab youth in Jordan. The results of this study, which is the first of its kind to be conducted in an Arab country, confirm the importance of enhancing suicide awareness and improving psychological help-seeking attitudes by increasing the levels of suicide literacy and decreasing the levels of stigma towards suicide and mental illness among this age group.

The results indicated that the sample had very low suicide literacy levels. The literacy level of this sample was found to be the lowest compared to the findings of other studies which used the LOSS among different populations (e.g. public community and university students) in the following countries: Australia (Batterham et al., 2013a, 2013b), China (Han et al., 2017), Turkey (Öztürk \& Akin, 2018), and Jordan (Aldalaykeh et al., 2020). This could be explained by the fact that the participants in the current study were aged 16 24 years, with a mean age of 18.6 years, which is considered young. The young ages of the participants may have limited their knowledge about mental health issues and suicide (Ahmed \& Baruah, 2017). Moreover, the majority of the participants had a lack of previous exposure to suicidal or mentally ill patients and very few participants had history or family history of mental illnesses or suicidal thoughts/attempts, which may have negatively affected their suicide literacy levels. However, the present study did not assess the associations of age and educational level with suicide literacy level among youths, which highlights the need for further research.
Table 4 Hierarchical Regression: Attitudes towards Professional Psychological Help Seeking $(\mathrm{N}=707)$

\begin{tabular}{|c|c|c|c|c|}
\hline Model & $\beta$ & Adjusted $\mathrm{R}^{2}$ & $\mathrm{~F}$ & F Change \\
\hline 1 LOSS & $0.329 * * *$ & 0.140 & $39.31 * * *$ & $39.31 * * *$ \\
\hline SOSS-SF stigmatization & 0.033 & & & \\
\hline SOSS-SF glorification & $-0.119 *$ & & & \\
\hline 2 LOSS & $0.271 * * *$ & 0.188 & $19.10 * * *$ & $7.85 * * *$ \\
\hline SOSS-SF stigmatization & 0.050 & & & \\
\hline SOSS-SF glorification & $-0.109 *$ & & & \\
\hline Gender & $-0.206 * * *$ & & & \\
\hline Being a student & -0.027 & & & \\
\hline Health insurance & -0.027 & & & \\
\hline Mother's employment status & 0.020 & & & \\
\hline Mental illness & 0.049 & & & \\
\hline Previous suicidal ideation or suicide attempt & 0.062 & & & \\
\hline
\end{tabular}


In addition, Jordan has no active anti-stigma or mental health awareness campaigns, limited mental health services, and limited numbers of mental health professionals (e.g., psychologists and counsellors), which may have contributed to the low levels of suicide literacy and negative attitudes revealed in the current study (Aldalaykeh et al., 2019). Another possible explanation is that the sample had high levels of stigma towards suicidal people and negative attitudes towards seeking psychological help, which may have also negatively impacted their suicide literacy levels. The results revealed that suicide literacy had a significant negative association with the stigma subscale of the SOSS-SF and a significant positive association with attitudes towards seeking psychological help among the sample. These relationships indicate that individuals who have low levels of suicide literacy are more likely to report higher levels of suicide stigma and more negative attitudes towards seeking psychological help. This latter statement needs further research due to this study being cross-sectional, we were unable to assess the causal relationship between suicide literacy and suicide stigma and attitudes towards seeking psychological help among our sample.

With regard to suicide stigma, the results indicated that the participants had relatively high levels of stigma towards suicidal people. This finding is consistent with the findings of a Jordanian study conducted on a sample of university students (Aldalaykeh et al., 2020) and is somewhat similar to the findings of studies conducted in Turkey (Öztürk \& Akin, 2018), China (Han et al., 2017), and Qatar (Zolezzi et al., 2017). On the other hand, a study conducted in Australia reported lower suicide stigma levels among the public community and among university students (Batterham et al., 2013a, 2013b). The high levels of stigma towards suicidal people among our sample may be attributed to Arab culture. Most Arabs are conservative within their daily lives in Arab communities and they prefer not to share their private matters, such as their mental health problems, with healthcare professionals (Al Ali et al., 2017; Ismayilova et al., 2013; Rayan \& Jaradat, 2016; Zolezzi et al., 2017). In turn, this may impact their attitudes towards psychological help-seeking and may lead them to stigmatize mental illnesses, mental illness treatments, and mental illness services (Al Ali et al., 2017; Ismayilova et al., 2013; Rayan \& Jaradat, 2016). The results also supported this, as $19.1 \%(n=$ 135) of the participants reported that they would not be willing to seek psychological help, as their preferred source of psychological help was 'no one'. Another explanation is that there was a significant negative correlation between suicide stigma and suicide literacy among the sample, with high levels of suicide stigma associated with lower levels of suicide literacy. This finding needs further research to assess the change in stigma and attitudes and the effect of suicide literacy on stigma and attitudes. Due to this study being cross-sectional, we were unable to assess the causal relationship between suicide literacy and stigma among our sample. Previous studies have highlighted the important impact of improving literacy on decreasing stigmatizing attitudes (Abuhammad \& Hamaideh, 2021; Batterham et al., 2013a, 2013b; Öztürk \& Akin, 2018). For example, Abuhammad \& Hamaideh, 2021) reported that Jordanian university students' attitudes towards mental illness changed positively after the students attended an educational course on mental health.

As for help-seeking attitudes, the results indicated relatively negative attitudes towards seeking psychological help among the sample. This result is consistent with the results of previous studies in Jordan and other Arab countries, which have indicated negative attitudes towards mental illness and mental health services (Al Ali et al., 2017; Aldalaykeh et al., 2019; Dardas et al., 2019; Rayan \& Jaradat, 2016). In comparison, studies conducted in Western countries usually indicate more positive attitudes towards mental illness and mental health services (Aldalaykeh, 2016). The results related to the participants' preferred sources of psychological help also indicated that the participants had negative attitudes towards seeking psychological help, as the majority of the participants preferred informal psychological help sources over formal sources. The most frequently reported source was family members, relatives, and friends, whilst the least frequently reported source was mental healthcare professionals, such as psychiatrists. Our results are in line with the results of previous studies conducted in Jordan (Al Ali et al., 2017; Dardas et al., 2019), in Qatar (Zolezzi et al., 2017), and in United Arab Emirates (Amiri et al., 2013). Arab people have been found to believe that informal sources of psychological help, such as seeking help from others who have similar experiences or viewpoints, are the easiest, quickest, and least stigmatized form of treatment (Al Ali et al., 2017; Dardas \& Simmons, 2015). These results may indicate that in Arab culture, family and society play an important role in helping the individual deal with psychological or mental health problems (Dardas et al., 2019). The low suicide literacy levels among the sample may also explain these results. Previous studies have found that the individual's level of literacy about a given disease or condition may affect that individual's help-seeking attitudes and behaviors (Ahmed \& Baruah, 2017; Batterham et al., 2013b; Darraj et al., 2016). Further, the participants' young ages may have also negatively affected their literacy levels and their attitudes towards seeking psychological help (Alonzo et al., 2011). This is supported by previous studies which have found a negative relationship between age and help-seeking attitudes (Daniels \& Jung, 2009; Graff et al., 2008). Lastly, the average family size among the sample was six members, which is considered large and may have negatively impacted the participants' attitudes towards seeking psychological help (Fung et al., 2008). Individuals who belong to large families may be impacted by their family members' negative attitudes towards mental illnesses and psychological help-seeking (Fung et al., 2008). 
As with regard to the predictors of the participants' attitudes towards seeking psychological help, the results of the regression analysis indicated that suicide literacy was the strongest predictor of help seeking attitudes among the sample. This result highlights the importance of holding suicide, mental illness, and mental health services awareness campaigns for youths in their schools and universities in order to increase their literacy levels and hence change their psychological help-seeking attitudes. Gender was the second strongest predictor of psychological help-seeking attitudes. Males were found to have more negative attitudes towards psychological help-seeking than females. This finding is consistent with previous Western studies (Batterham et al., 2013b) and inconsistent with previous Arabic studies which have reported no significant association between gender and attitudes towards suicide and mental illness (Amiri et al., 2013; Zolezzi et al., 2017). Further, the fact that the majority of the participants in the current study were male may explain the participants' negative attitudes towards seeking psychological help. Lastly, the SOSS-SF glorification subscale was the third strongest predictor of psychological help-seeking attitudes among the sample, and it was found to have a negative effect. This indicates that controlling young people's glorification or normalization of suicide attempts or self-harm acts through awareness campaigns about suicide may improve their psychological help-seeking attitudes. Interestingly, the results showed that both the stigma and glorification subscales of the SOSS-SF were not major predictors of psychological help-seeking attitudes among the sample. Overall, more attention should be paid towards increasing suicide literacy levels and decreasing the levels of stigma towards mental health issues in order to improve help-seeking attitudes among this age group (Dardas et al., 2019). However, the results of this study need to be verified by further studies among other age groups in Jordan and in other Arabic countries.

\section{Limitations}

There were several limitations to the current study. First, the use of an online survey and snowball sampling methods may have led to selection bias, as only young people who have access to the internet and the study link had a chance to participate in this study. Second, the use of a cross-sectional design provided information about the levels and the relationships between the study variables without causal inferences. Third, the participants' responses to the self-report instruments used to measure stigma and attitudes may have been influenced by social desirability. Fourth, there may be factors which were not assessed in this study, but which may have impacted the participants' suicide literacy, such as income, parents' educational level, religion, living arrangements, access to the internet, and social media. Therefore, further studies which examine these factors and their associations with suicide literacy, suicide stigma, and psychological helpseeking attitudes among youths are recommended. Finally, data were collected during the COVID-19 pandemic, which may have impacted the results as the pandemic is considered a contextual factor in terms of examining and addressing mental health issues among youths during this difficult time (Tanhan, 2020; Tanhan et al., 2020). However, despite these limitations, this study provides valuable information on suicide literacy, suicide stigma, and psychological help-seeking attitudes among Arab youth. Further, our findings need to be verified by future studies which use qualitative and longitudinal designs on different populations and representative study samples.

\section{Implications}

This study also has several important implications. The results of this study highlight the importance of developing and implementing awareness campaigns and educational programs for youth in schools and universities. Such interventions require strong support from and collaboration between governmental, political, and educational institutions. Therefore, our results may also help nursing leaders and healthcare institutions to improve mental health educational programs, plans, and services that are already existed to support community's mental health needs. Their efforts need to be directed towards demonstrating the required changes for effectively integrating mental health services in educational settings, including schools and universities. In addition, longitudinal and experimental studies which test the effectiveness of educational programs or interventions on suicide and mental illness among this age group are recommended. Such studies may help to establish causal relationships and identify the risk factors associated with suicide literacy, suicide stigma, and psychological help-seeking attitudes among youth. Another example is conducting online photovoice (OPV) as a qualitative method for understanding and addressing mental health issues among youth from a contextual perspective (Tanhan \& Strack, 2020). Lastly, this study can be replicated among this age group in other Arab countries as well as among other age groups in Jordan in order to increase the generalizability of the study results.

In conclusion, our results showed very low suicide literacy levels, high suicide stigma levels, and negative attitudes towards seeking psychological help among a sample of Arab youth. These results could be related to most of the participants had a lack of previous exposure to suicidal or mentally ill patients as well as their young ages may have limited their knowledge about suicide and mental health issues. Thus, healthcare leaders, professionals, and researchers should be aware of the current evidence regarding suicide and attitudes towards seeking professional psychological help among youth within the context of their society. All interventions and 
support should be culturally consistent with the Arab beliefs and views of mental health. The results of this study confirm the presence of an urgent need for designing and implementing educational programs and awareness campaigns on suicide and mental illness among youth in schools and universities. Such interventions would increase levels of suicide and mental health literacy, decrease levels of stigma towards suicide and mental illness, change psychological help-seeking attitudes, and hence improve the use of mental health services and prevent suicide and mental illness among this age group in Arabic countries like Jordan. Further research on suicide awareness and psychological help-seeking attitudes is urgently needed at different levels, including family and community levels, to address the mental health needs of this vulnerable population.

\section{Acknowledgements Not applicable.}

Authors' Contributions YA conceived of the study and its design and prepared the study material; YA and MA performed the data collection; YA and MA performed the statistical analysis and wrote the findings; MA and YA discussed the analysis and participated in interpretation of findings; YA wrote the first draft of the manuscript. Both authors reviewed and commented on previous versions of the manuscript. Both authors read and approved the final manuscript.

Funding No funding was received for conducting this study.

\section{Declarations}

Ethical Approval All procedures performed in this study involving human participants were in accordance with the ethical standards of University's Research Ethics Board and with the 1975 Helsinki Declaration. The study was approved by the institutional review board of the Jordan University of Science and Technology (No. 164/132/2020).

Consent for Publication Not applicable.

Data Sharing and Declaration Data will be made available on request.

Informed Consent Informed consent was electronically obtained from all participants included in the study before participation.

Conflict of Interest The authors declare that they have no conflict of interest.

\section{References}

Abuhammad, S., \& Hamaideh, S. (2021). Nursing Students' attitudes toward seeking professional psychological help before and after attending a mental health course. Nursing Education Perspectives. https://doi.org/10.1097/01.NEP.0000000000000796, Publish Ahead of Print

Ahmed, N., \& Baruah, A. (2017). Awareness about mental illness among the family members of persons with mental illness in a selected
District of Assam. Indian Journal of Social Psychiatry, 33(2), 171. https://doi.org/10.4103/0971-9962.209199.

Aldalaykeh, M. (2016). Predictors of Partial Hospitalization Attendance by U.S. Adults with Mental Illness. Kent State University. Retrieved from https://etd.ohiolink.edu/pg_10?0::NO:10:P10_ACCESSION NUM:kent1468621382

Aldalaykeh, M., Al-Hammouri, M. M., \& Rababah, J. (2019). Predictors of mental health services help-seeking behavior among university students. Cogent Psychology., 6, 1660520. https://doi.org/10.1080/ 23311908.2019.1660520.

Aldalaykeh, M., Dalky, H., Shahrour, G., \& Rababa, M. (2020). Psychometric properties of two Arabic suicide scales: Stigma and literacy. Heliyon, 6(4), e03877. https://doi.org/10.1016/j.heliyon. 2020.e03877.

Alonzo, D. M., Harkavy-Friedman, J. M., Stanley, B., Burke, A., Mann, J. J., \& Oquendo, M. A. (2011). Predictors of treatment utilization in major depression. Archives of Suicide Research, 15(2), 160-171. https://doi.org/10.1080/13811118.2011.566052.

Al Ali, N. M., Alqurneh, M. K., Dalky, H., \& Al-Omari, H. (2017). Factors affecting help-seeking attitudes regarding mental health services among attendance of primary health care centers in Jordan. International Journal of Mental Health, 46, 38-51. https://doi.org/ 10.1080/00207411.2016.1264039.

Al-Shannaq, Y., Mohammad, A. A., \& Aldalaykeh, M. (2021). Depression, coping skills, and quality of life among Jordanian adults during the initial outbreak of COVID-19 pandemic: Cross sectional study. Heliyon, 7(4), e06873. https://doi.org/10.1016/j.heliyon. 2021.e06873.

Amiri, L., Voracek, M., Yousef, S., Galadari, A., Yammahi, S., Sadeghi, M. R., ... \& Dervic, K. (2013). Suicidal behavior and attitudes among medical students in the United Arab Emirates. Crisis, 34(2): 116-123. 33. https://doi.org/10.1027/0227-5910/a000170

Amri, S., \& Bemak, F. (2013). Mental health help-seeking behaviors of Muslim immigrants in the United States: Overcoming social stigma and cultural mistrust. Journal of Muslim Mental Health, 7(1). https:// doi.org/10.3998/jmmh.10381607.0007.104.

Arafat, S. Y., Al Mamun, M. A., \& Uddin, M. S. (2019). Depression literacy among first-year university students: A cross-sectional study in Bangladesh. Global Psychiatry, 2(1), 31-36. https://doi.org/10. 2478/gp-2019-0002.

Batterham, P. J., Calear, A. L., \& Christensen, H. (2013a). The stigma of suicide scale. Crisis, 34(1), 13-21. https://doi.org/10.1027/02275910/a000156.

Batterham, P. J., Calear, A. L., \& Christensen, H. (2013b). Correlates of suicide stigma and suicide literacy in the community. Suicide and Life-threatening Behavior, 43(4), 406-417. https://doi.org/10.1111/ sltb. 12026.

Bronfenbrenner, U. (1977). Toward an experimental ecology of human development. American Psychologist, 32, 513-531. https://doi.org/ 10.1037/0003-066X.32.7.513.

Bronfenbrenner, U., \& Evans, G. W. (2000). Developmental science in the 21st century: Emerging questions, theoretical models, research designs and empirical findings. Social Development, 9(1), 115-125. https://doi.org/10.1111/1467-9507.00114.

Calear, A. L., Batterham, P. J., \& Christensen, H. (2014). Predictors of help-seeking for suicidal ideation in the community: Risks and opportunities for public suicide prevention campaigns. Psychiatry Research, 219(3), 525-530. https://doi.org/10.1016/j.psychres. 2014.06.027.

Choudhry, F. R., Mani, V., Ming, L. C., \& Khan, T. M. (2016). Beliefs and perception about mental health issues: A meta-synthesis. Neuropsychiatric Disease and Treatment, 12, 2807-2818. https:// doi.org/10.2147/ndt.s111543.

Clement, S., Schauman, O., Graham, T., Maggioni, F., Evans-Lacko, S., Bezborodovs, N., et al. (2015). What is the impact of mental healthrelated stigma on help-seeking? A systematic review of quantitative 
and qualitative studies. Psychological Medicine, 45(1), 11-27. https://doi.org/10.1017/s0033291714000129.

Ciftci, A., Jones, N., \& Corrigan, P. W. (2013). Mental health stigma in the Muslim community. Journal of Muslim Mental Health, 7(1). https://doi.org/10.3998/jmmh.10381607.0007.102.

Daniels, M., \& Jung, S. (2009). Missed initial appointments at an outpatient forensic psychiatric clinic. The Journal Of Forensic Psychiatry And Psychology (Print), 20(6), 964-973. https://doi.org/10.1080/ 14789940903174204.

Dardas, L. A., \& Simmons, L. A. (2015). The stigma of mental illness in Arab families: A concept analysis. Journal of Psychiatric and Mental Health Nursing, 22(9), 668-679. https://doi.org/10.1111/ jpm.12237.

Dardas, L. A., Silva, S., Noonan, D., \& Simmons, L. A. (2016). A pilot study of depression, stigma, and attitudes towards seeking professional psychological help among Arab adolescents. International Journal of Adolescent Medicine and Health, 30(4). https://doi.org/ 10.1515/ijamh-2016-0070.

Dardas, L. A., Silva, S., Noonan, D., \& Simmons, L. A. (2017). Studying depression among Arab adolescents: Methodological considerations, challenges, and lessons learned from Jordan. Stigma and Health, 3(4), 296-304. https://doi.org/10.1037/sah0000101.

Dardas, L. A., Silva, S. G., van de Water, B., Vance, A., Smoski, M. J., Noonan, D., \& Simmons, L. A. (2019). Psychosocial correlates of Jordanian adolescents' help-seeking intentions for depression: Findings from a nationally representative school survey. The Journal of School Nursing, 35(2), 117-127. https://doi.org/10. 1177/1059840517731493.

Darraj, H. A., Mahfouz, M. S., Sanosi, M. R., Al Badedi, M., Sabai, A., et al. (2016). Arabic translation and psychometric evaluation of the depression literacy questionnaire among adolescents. Psychiatry Journal, 8045262, 1-7. https://doi.org/10.1155/2016/8045262.

El Halabi, S., El Hayek, R., Kahil, K., Nofal, M., \& El Hayek, S. (2020). Characteristics of attempted suicide in the Middle East and North Africa region: The mediating role of Arab culture and religion. Mediterranean Journal of Emergency Medicine \& Acute Care, 1(3).

Eskin, M., Kujan, O., Voracek, M., Shaheen, A., Carta, M. G., Sun, J. M., Flood, C., Poyrazli, S., Janghorbani, M., Yoshimasu, K., Mechri, A., Khader, Y., Aidoudi, K., Bakhshi, S., Harlak, H., Ahmead, M., Moro, M. F., Nawafleh, H., Phillips, L., Abuderman, A., Tran, U. S., \& Tsuno, K. (2016). Cross-national comparisons of attitudes towards suicide and suicidal persons in university students from 12 countries. Scandinavian Journal of Psychology, 57(6), 554563. https://doi.org/10.1111/sjop.12318.

Eskin, M., Poyrazli, S., Janghorbani, M., Bakhshi, S., Carta, M. G., Moro, M. F., Tran, U. S., Voracek, M., Mechri, A., Aidoudi, K., Hamdan, M., Nawafleh, H., Sun, J. M., Flood, C., Phillips, L., Yoshimasu, K., Tsuno, K., Kujan, O., Harlak, H., Khader, Y., Shaheen, A., \& Taifour, S. (2019). The role of religion in suicidal behavior, attitudes and psychological distress among university students: A multinational study. Transcultural Psychiatry, 56(5), 853-877. https://doi. org/10.1177/1363461518823933.

Eskin, M., Baydar, N., El-Nayal, M., Asad, N., Noor, I. M., Rezaeian, M., et al. (2020). Associations of religiosity, attitudes towards suicide and religious coping with suicidal ideation and suicide attempts in 11 muslim countries. Social Science \& Medicine, 265, 113390. https://doi.org/10.1016/j.socscimed.2020.113390.

Fekih-Romdhane, F., Tounsi, A., Rejeb, R. B., \& Cheour, M. (2020). Is religiosity related to suicidal ideation among Tunisian Muslim youth after the January 14th revolution? Community Mental Health Journal, 56(1), 165-173. https://doi.org/10.1007/s10597-01900447-z.

Fischer, E. H., \& Farina, A. (1995). Attitudes toward seeking professional psychologial help: A shortened form and considerations for research. Journal of College Student Development, 36(4), 368-373. https://doi.org/10.1037/t05375-000.
Fung, K., Tsang, H., \& Corrigan, P. (2008). Self-stigma of people with schizophrenia as predictor of their adherence to psychosocial treatment. Psychiatric Rehabilitation Journal, 32(2), 95-104. https://doi. org/10.2975/32.2.2008.95.104.

Graff, F., Griffin, M., \& Weiss, R. (2008). Predictors of dropout from group therapy among patients with bipolar and substance use disorders. Drug and Alcohol Dependence, 94, 272-275. https://doi.org/ 10.1016/j.drugalcdep.2007.11.002.

Han, J., Batterham, P. J., Calear, A. L., Wu, Y., Shou, Y., \& van Spijker, B. A. J. (2017). Translation and validation of the Chinese versions of the suicidal ideation attributes scale, stigma of suicide scale, and literacy of suicide scale. Death Study, 41(3), 173-179. https://doi. org/10.1080/07481187.2016.1214633.

Ismayilova, L., Hmoud, O., Alkhasawneh, E., Shaw, S., \& El- Bassel, N. (2013). Depressive symptoms among Jordanian youth: Results of a national survey. Community Mental Health Journal, 49, 133-140. https://doi.org/10.1007/s10597-012-9529-7.

Keller, S., McNeill, V., Honea, J., \& Paulson Miller, L. (2019). A look at culture and stigma of suicide: Textual analysis of community theatre performances. International Journal of Environmental Research and Public Health, 16(3), 352. https://doi.org/10.3390/ ijerph16030352.

Koenig, H. G., Al Zaben, F., \& Khalifa, D. A. (2012). Religion, spirituality and mental health in the west and the Middle East. Asian Journal of Psychiatry, 5(2), 180-182. https://doi.org/10.1016/j.ajp. 2012.04.004.

Koenig, H. G., \& Al Shohaib, S. S. (2019). Religiosity and mental health in Islam. Islamophobia and psychiatry, 55-65. https://doi.org/10. 1007/978-3-030-00512-2 5.

Lawrence, R. E., Oquendo, M. A., \& Stanley, B. (2016). Religion and suicide risk: A systematic review. Archives of Suicide Research, 20(1), 1-21. https://doi.org/10.1080/13811118.2015.1004494.

Lester, D., \& Walker, R. L. (2017). Religiosity is a protective factor for suicidal ideation in European American students but not in African American students. OMEGA-Journal of Death and Dying, 74(3), 295-303. https://doi.org/10.1177/0030222815598452.

Liu, X., Huang, Y., \& Liu, Y. (2018). Prevalence, distribution, and associated factors of suicide attempts in young adolescents: Schoolbased data from 40 low-income and middle-income countries. PLoS One, 13(12), e0207823. https://doi.org/10.1371/journal. pone.0207823.

Mahfoud, Z. R., Afifi, R. A., Haddad, P. H., \& DeJong, J. (2011). Prevalence and determinants of suicide ideation among Lebanese adolescents: Results of the GSHS Lebanon 2005. Journal of Adolescence, 34(2), 379-384. https://doi.org/10.1016/j. adolescence.2010.03.009.

Madge, N., Hewitt, A., Hawton, K., Wilde, E. J. D., Corcoran, P., Fekete, S., Heeringen, K. ., Leo, D. D., \& Ystgaard, M. (2008). Deliberate self-harm within an international community sample of young people: Comparative findings from the Child \& Adolescent Self-harm in Europe (CASE) study. Journal of Child Psychology and Psychiatry, 49(6), 667-677. https://doi.org/10.1111/j.1469-7610. 2008.01879.x.

Morad, M., Merrick, E., Schwarz, A., \& Merrick, J. (2005). A review of suicide behavior among Arab adolescents. The Scientific World Journal, 5, 674-679. https://doi.org/10.1100/tsw.2005.84.

Niederkrotenthaler, T., Reidenberg, D. J., Till, B., \& Gould, M. S. (2014). Increasing help-seeking and referrals for individuals at risk for suicide by decreasing stigma: The role of mass media. American Journal of Preventive Medicine, 47(3), S235-S243. https://doi.org/ 10.1016/j.amepre.2014.06.010.

Okasha, A., Karam, E., \& Okasha, T. (2012). Mental health services in the Arab world. World Psychiatry, 11(1), 52-54. https://doi.org/10. 1016/j.wpsyc.2012.01.008.

Öztürk, A., \& Akin, S., (2018). Evaluation of knowledge level about suicide and stigmatizing attitudes in university students toward 
people who commit suicide. Journal of Psychiatric Nursing, 9(2), 96-104. Doi: https://doi.org/10.14744/phd.2018.49389.

Pandey, A. R., Bista, B., Dhungana, R. R., Aryal, K. K., Chalise, B., \& Dhimal, M. (2019). Factors associated with suicidal ideation and suicidal attempts among adolescent students in Nepal: Findings from global school-based students health survey. PLoS One, 14(4), e0210383. https://doi.org/10.1371/journal.pone.0210383.

Peel, R., Buckby, B., \& McBain, K. A. (2017). Comparing the effect of stigma on the recognition of suicide risk in others between Australia and Brazil. GSTF Journal of Psychology, 3(2), 1-10. https://doi.org/ 10.5176/2345-7872 3.2 43.

Ram, D., Chandran, S., \& Basavana, G. H. (2017). Suicide and depression literacy among healthcare profession students in tertiary care center in South India. Psychiatry and Behavioral Sciences, 7(3), 149-155. https://doi.org/10.5455/jmood.20170830064910.

Rayan, A., \& Jaradat, A. (2016). Stigma of mental illness and attitudes toward psychological help-seeking in Jordanian University students. Research in Psychology and Behavioral Sciences, 4(1), 7-14. Doi: https://doi.org/10.12691/rpbs-4-1-2.

Rockett, I. R., Regier, M. D., Kapusta, N. D., Coben, J. H., Miller, T. R., Hanzlick, R. L., et al. (2012). Leading causes of unintentional and intentional injury mortality: United States, 2000-2009. American Journal of Public Health, 102(11), e84-e92. https://doi.org/10. 2105/ajph.2012.300960.

Shah, A., \& Chandia, M. (2010). The relationship between suicide and Islam: A cross-national study. Journal of Injury and Violence Research, 2(2), 93.-97. https://doi.org/10.5249/jivr.v2i2.60.

Tanhan, A. (2019). Acceptance and commitment therapy with ecological systems theory: Addressing Muslim mental health issues and wellbeing. Journal of Positive Psychology and Wellbeing, 3(2), 197-219. https://doi.org/10.47602/jpsp.v3i2.172.

Tanhan, A. (2020). Covid-19 sürecinde online seslifoto (OSF) yöntemiyle biyopsikososyal manevi ve ekonomik meseleleri ve genel iyi oluș düzeyini ele almak: OSF'nin Türkçeye uyarlanması. [utilizing online photovoice (OPV) methodology to address biopsychosocial spiritual economic issues and wellbeing during covid-19: Adapting OPV to Turkish.], Turkish studies, 15(4), https://doi.org/10.7827/TurkishStudies.44451, Volume 15 Issue 4, 1029, 1086

Tanhan, A., \& Francisco, V. T. (2019). Muslims and mental health concerns: A social ecological model perspective. Journal of Community Psychology, 47(4), 964-978. https://doi.org/10.1002/jcop.22166.

Tanhan, A., \& Strack, R. W. (2020). Online photovoice to explore and advocate for Muslim biopsychosocial spiritual wellbeing and issues: Ecological systems theory and ally development. Current Psychology, 39(6), 2010-2025. https://doi.org/10.1007/s12144020-00692-6.

Tanhan, A., Yavuz, K. F., Young, J. S., Nalbant, A., Arslan, G., Yıldırım, M., ... \& Çiçek, İ. (2020). A proposed framework based on literature review of online contextual mental health services to enhance wellbeing and address psychopathology during COVID-19. Electronic Journal of General Medicine, 17(6), em254. https://doi. org/10.31219/osf.io/4wbd8

United Nations. Definition of youth. (2020). Retrieved from: https:// www.un.org/en/sections/issues-depth/youth-0/

World Health Organization. (2018). Suicide rate estimates, crude, 15-29 and 30-49 years Estimates by country. Retrieved from: https://apps. who.int/g ho/d a t a / n o d e.main. MH S U I C I D E A GEGROUPS15293049? ?ang=en

World Health Organization. (2019). Suicide. Retrieved from: https:// www.who.int/news-room/fact-sheets/detail/suicide

Wu, A., Wang, J. Y., \& Jia, C. X. (2015). Religion and completed suicide: A meta-analysis. PLoS One, 10(6), e0131715. https://doi.org/10. 1371/journal.pone. 0131715 .

Zolezzi, M., Bensmail, N., Zahrah, F., Khaled, S. M., \& El-Gaili, T. (2017). Stigma associated with mental illness: Perspectives of university students in Qatar. Neuropsychiatric Disease and Treatment, 13, 1221-1233. https://doi.org/10.2147/NDT.S132075.

Publisher's Note Springer Nature remains neutral with regard to jurisdictional claims in published maps and institutional affiliations. 